\title{
Diagnostic criteria for gestational diabetes: Who decides?
}

\author{
Edmond A. Ryan MD
}

See related research article by Retnakaran and colleagues on page 1353 and at www.cmaj.ca/lookup/doi/10.1503/cmaj.111154

$\mathrm{W}$ hen we hear of an 11-pound baby being delivered, maternal diabetes comes to mind. This link between the mother's glucose levels and macrosomia provides the main rationale for treating gestational diabetes. Recently proposed criteria for gestational diabetes will result in the condition being diagnosed in nearly a fifth of all pregnancies. ${ }^{1}$ In a related article in CMAJ, Retnakaran and colleagues report that, among women who did not have gestational diabetes according to current diagnostic criteria, impaired glucose tolerance was not a significant independent predictor of having a large-for-gestational-age infant. ${ }^{2}$ Given that many of these women would be labelled as having gestational diabetes using the proposed diagnostic criteria, ${ }^{1}$ this should give us pause before accepting such changes to the criteria.

Many studies have shown an association between macrosomia and maternal diabetes. The Hyperglycemia and Adverse Pregnancy Outcome (HAPO) Study $(n=23$ 316) showed a continuous association between increased infant birth weight and maternal glucose levels below those diagnostic of diabetes. ${ }^{3}$ In 1952, Pedersen hypothesized that excess maternal glucose crosses the placenta and leads to fetal hyperglycemia, which in turn leads to hyperinsulinemia and excessive fetal growth. ${ }^{4}$ In large-for-gestational-age infants of women with diabetes, the excess weight is not simply from the glucose being turned into fat, but many internal organs are enlarged as well. Currently, the best explanation may be that an elevated fetal insulin level downregulates insulin growth factor (IGF)binding protein-1, which allows more unbound IGF-1 to promote excess growth.

Factors other than maternal glucose levels are recognized to play a role in predicting birth weight: the mother's body mass index (BMI), her weight gain during pregnancy, the height of the father, the baby's sex, the duration of the pregnancy, the mother's smoking status and her ethnicity are some of the known principal players. Of these, the factor most amenable to change is the glucose level, which explains the focus on diabetes and gestational diabetes in trying to prevent macrosomia and its attendant adverse complications (e.g., shoulder dystocia, birth trauma and cesarean delivery).
Retnakaran and colleagues present useful information about 472 women and factors contributing to their offspring's birth weight. The women studied did not have gestational diabetes, as determined from a 100-g oral glucose tolerance test and defined according to the National Diabetes Data Group criteria. ${ }^{5}$

In their logistic regression analysis, the positive independent metabolic predictors of having a large-for-gestational-age infant were maternal BMI before pregnancy (odds ratio [OR] 1.16, 95\% confidence interval [CI] $1.05-1.27$, per $1 \mathrm{~kg} / \mathrm{m}^{2}$ increase) and weight gain during pregnancy up to the time of the oral glucose tolerance test (OR $1.12,95 \%$ CI $1.05-1.19$, per $1 \mathrm{~kg}$ increase); the maternal leptin level was a negative predictor (OR $0.5,95 \%$ CI $0.30-0.82$ ). Of note, the factors associated with changes in birth weight could explain only $26 \%$ of the variance in birth weight. The association between leptin and macrosomia is counterintuitive. Usually leptin increases with BMI, so the negative association between leptin and having a large-for-gestational-age infant is striking and is testimony to the complexity of issues surrounding control of birth weight.

The large HAPO study showed that the association between maternal glucose levels and birth weight is a continuum with no clear threshold. ${ }^{3}$ The International Association of Diabetes and Pregnancy Study Groups came to a consensus in choosing diagnostic thresholds of 5.1, 10.0 and 8.5 $\mathrm{mmol} / \mathrm{L}$ for fasting, one-hour and two-hour plasma glucose concentrations, respectively, on a single 75-g oral glucose tolerance test. ${ }^{1}$ These thresholds are based on the glucose levels associated with a 1.75 -fold increased risk of having a large-forgestational-age infant based on data from the HAPO study. The Canadian Diabetes Associa-
Competing interests: Edmond Ryan received research grants from Pfizer for studies into inhaled insulin use as treatment in general diabetes and from Novo Nordisk Canada for detemir insulin use in type 1 diabetes in pregnancy; he is on the Canadian Diabetes Association subcommittee for diabetes in pregnancy to prepare 2013 guidelines; he attended a planning committee meeting for the upcoming National Institutes of Health consensus conference on diagnosing gestational diabetes in October 2012 and will be speaking at the conference.

This article was solicited and has not been peer reviewed.

Correspondence to: Edmond Ryan, edmond.ryan@ualberta.ca

CMAJ 2012. DOI:10.1503 /cmaj.120682 
tion's current guidelines for diagnosing gestational diabetes use higher thresholds for glucose levels that are virtually identical to those associated with a 2-fold increased risk of a large-for-gestationalage infant based on the HAPO data. ${ }^{6}$

With the stroke of a consensus pen, nearly onefifth of pregnant women - more than double the current incidence in Canada - would be labelled as having gestational diabetes if the criteria of the International Association of Diabetes and Pregnancy Study Groups were to be adopted.

Further analysis of HAPO data showed maternal BMI to be at least as important as glucose levels for predicting birth weight. ${ }^{7.8}$ In the report by Retnakaran and colleagues, among the additional women who would be identified as having gestational diabetes if the lower diagnostic thresholds were used, maternal BMI before pregnancy, weight gain during pregnancy and leptin levels not the mother's glucose level - were independently predictive of birth weight. ${ }^{2}$

A recent analysis of the new criteria indicates that they are cost-effective only if the diagnosis of gestational diabetes can be linked to a future reduction of type 2 diabetes. ${ }^{9}$

In the United States, the American Diabetes Association has endorsed the new criteria, ${ }^{10}$ but the American College of Obstetrics and Gynecology has deferred a decision of whether to update its diagnostic criteria until after a $\mathrm{Na}-$ tional Institutes of Health consensus conference on the topic, to be held in October 2012.

In Canada, the Canadian Diabetes Association has yet to issue updated guidelines, but the province of British Colombia has adopted the new criteria. A pregnant woman in $\mathrm{BC}$ who has a glucose level of $10.0 \mathrm{mmol} / \mathrm{L}$ at one hour on a single 75 -g oral glucose tolerance test at 25 weeks' gestation will be told she has gestational diabetes. She will be referred to a diabetes clinic, given a diet to follow, instructed to monitor her glucose levels, undergo more fetal obstetric monitoring, likely will be delivered early with increased risk of cesarean delivery and may well encounter higher premiums if she seeks life insurance in the future. Her counterpart in Alberta with the same result on the oral glucose tolerance test will be told all is well. This raises issues in any health care system, particularly a publicly funded one such as ours that has finite funds.

So, who decides if the label of gestational diabetes is medically justified, if making the diagnosis is worth it, or if our health care system can afford it? Who integrates these factors and balances the payment for this extra work when other areas may be more deserving? In the area of gestational diabetes, the medical justification for expanding the diagnostic criteria is certainly debatable. The proposed criteria failed to pass muster in the first cost-benefit analysis.

The upcoming US National Institutes of Health's consensus conference on diagnosing gestational diabetes will use an established process: a panel with no vested interest in the disease will hear the evidence from independent analyses as well as opinions from proponents for various positions and give their considered judgment. If we are not going to invest in some similar analytical process in Canada, perhaps we should heed the verdict from the consensus conference.

Maternal weight and glucose levels both play a role in large-for-gestational-age infants, but their respective contributions need to be defined. In the meantime, the concluding comments of Retnakaran and colleagues - to target weight and weight gain in pregnant women to prevent excess fetal growth - are sound. Evidence from a pilot project in Australia showed that a simple intervention for obese women that included a brief session with a nutritionist at each antenatal visit ameliorated weight gain in pregnancy and reduced the incidence of gestational diabetes. ${ }^{11}$ Evaluating such strategies while researchers determine what really causes large babies seems a more prudent way to spend money.

\section{References}

1. Metzger BE, Gabbe SG, Persson B, et al. International Association of Diabetes and Pregnancy Study Groups recommendations on the diagnosis and classification of hyperglycemia in pregnancy. Diabetes Care 2010;33:676-82.

2. Retnakaran R, Ye C, Hanley AJG, et al. Effect of maternal weight, adipokines, glucose intolerance and lipids on infant birth weight among women without gestational diabetes mellitus. CMAJ 2012;184:1353-60.

3. Metzger BE, Lowe LP, Dyer AR, et al. Hyperglycemia and adverse pregnancy outcomes. N Engl J Med 2008;358:1991-2002.

4. Pedersen J. Diabetes and pregnancy: blood sugar of newborn infants [PhD thesis]. Copenhagen [Denmark]: Danish Science Press; 1952. p. 230.

5. National Diabetes Data Group. Classification and diagnosis of diabetes mellitus and other categories of glucose intolerance. Diabetes 1979;28:1039-57.

6. Canadian Diabetes Association Clinical Practice Guidelines Expert Committee. Canadian Diabetes Association 2008 clinical practice guidelines for the prevention and management of diabetes in Canada. Can J Diab 2008;32(Suppl 1):S168-80.

7. HAPO Study Cooperative Research Group. Hyperglycaemia and Adverse Pregnancy Outcome (HAPO) Study: associations with maternal body mass index. BJOG 2010;117:575-84

8. Catalano PM, McIntyre HD, Cruickshank JK, et al.; HAPO Study Cooperative Research Group. The Hyperglycemia and Adverse Pregnancy Outcome Study: associations of GDM and obesity with pregnancy outcomes. Diabetes Care 2012;35:780-6.

9. Werner EF, Pettker CM, Zuckerwise L, et al. Screening for gestational diabetes mellitus: Are the criteria proposed by the International Association of the Diabetes and Pregnancy Study Groups cost-effective? Diabetes Care 2012;35:529-35.

10. American Diabetes Association. Diagnosis and classification of diabetes mellitus. Diab Care 2012;35(Suppl 1):S64-71.

11. Quinlivan JA, Lam LT, Fisher J. A randomized trial of a fourstep multidisciplinary approach to the antenatal care of obese pregnant women. Aust N Z J Obstet Gynaecol 2011;51:141-6.

Affiliation: Edmond Ryan is with the Division of Endocrinology and Metabolism, Department of Medicine, the Heritage Medical Research Centre and the Alberta Diabetes Institute, University of Alberta, Edmonton, Alta 